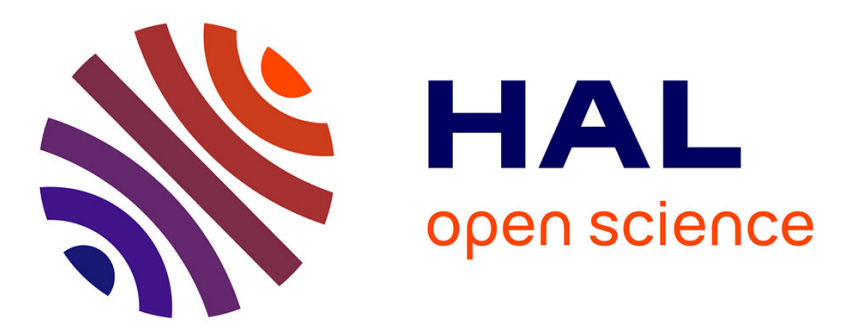

\title{
Surface plasmon polaritons locally excited on the ridges of metallic gratings
}

Bing Wang, Philippe Lalanne

\section{To cite this version:}

Bing Wang, Philippe Lalanne. Surface plasmon polaritons locally excited on the ridges of metallic gratings. Journal of the Optical Society of America. A, Optics and image science, 2010, 27 (6), pp.1432-1441. hal-00570610

\section{HAL Id: hal-00570610 \\ https://hal-iogs.archives-ouvertes.fr/hal-00570610}

Submitted on 5 Apr 2012

HAL is a multi-disciplinary open access archive for the deposit and dissemination of scientific research documents, whether they are published or not. The documents may come from teaching and research institutions in France or abroad, or from public or private research centers.
L'archive ouverte pluridisciplinaire HAL, est destinée au dépôt et à la diffusion de documents scientifiques de niveau recherche, publiés ou non, émanant des établissements d'enseignement et de recherche français ou étrangers, des laboratoires publics ou privés. 


\title{
Surface plasmon polaritons locally excited on the ridges of metallic gratings
}

\author{
B. Wang ${ }^{*, \dagger}$ and P. Lalanne \\ Laboratoire Charles Fabry de l'Institut d'Optique, CNRS, Univ. Paris-Sud, Campus Polytechnique, \\ 91127 Palaiseau cedex, France \\ *Corresponding author: wangb@imre.a-star.edu.sg
}

Received February 17, 2010; revised April 27, 2010; accepted April 27, 2010; posted April 28, 2010 (Doc. ID 124189); published May 21, 2010

\begin{abstract}
With the perspective to achieve an in-depth understanding of metallic periodic surfaces, we study the surface plasmon polaritons that are locally excited on the ridges (between the indentations) of metallic lamellar gratings composed of slits or grooves. An approximate model and fully vectorial computational results show that the normalized excitation rate is rather small for slit arrays $(\approx 10$ at maximum $)$ and is surprisingly weakly dependent on the metal permittivity. Additionally, the analysis is supported by an intuitive microscopic model that shines new light on the role of surface plasmons in the transmission and resonance anomalies of periodic metallic surfaces. (C) 2010 Optical Society of America
\end{abstract}

OCIS codes: $050.0050,290.0290,240.6680,310.6860$.

\section{INTRODUCTION}

With the recent advances in nanotechnology and in relation with the observation of the extraordinary optical transmission through arrays of tiny holes [1], the past decade has seen a renewed interest in exploiting the optical response of metallic materials with sub- $\lambda$ openings. Initial motivations were the trapping of light in small volumes [2], various applications of the extraordinary optical transmission [3,4], and waveguiding with lateral mode sizes well below the diffraction limit for ultra-compact surface-plasmon-polariton (SPP) photonic circuits $[5,6]$. This has initiated the creation of a variety of new and compact optical devices to manipulate SPPs.

Our current recipes to design these devices are based on the scattering of individual SPPs, which are assumed to be first generated by some illuminated apertures, then to propagate on the metal surface and to interact with nearby indentations before being recovered as freely propagating light or detected. However, the situation is not so simple; SPPs are only partly responsible for the electromagnetic interaction between adjacent openings on the metallic surface even at visible frequencies; other waves have to be considered for full assessment [7], and to add to the complexity, one has to take into consideration the inevitable cross-conversion between these waves and the SPPs [8].

Because of this difficulty, the SPP progression picture is often abandoned, and fully vectorial electromagnetic computations are preferably used for designing and analyzing sub- $\lambda$ metallic surfaces. The drawback of the computational approach is that our intuitive understanding is considerably lowered. This explains for instance why understanding the extraordinary optical transmission has led to a large controversy over the past ten years [9]. It is the authors' opinion that, although the transmission through $2 \mathrm{D}$ hole arrays is well documented nowadays, the role of SPPs in the transmission through periodic slit arrays is not yet fully understood-see the initial discussions in [10-12] and more recent analysis with the benefit of hindsight [13-18]. This is all the more surprising as these gratings, which have been initially developed for light spectroscopy, are presently becoming important components for various applications, such as polarization control [19,20], SPP-coupling [21], hot-spot generation [22], spectral filtering [23], on-chip optical information processing, nanoscience research [6], etc.

In order to bring new light on the scattering physics of sub- $\lambda$ slit arrays, we use here the theoretical formalism recently developed in [21] and study the SPPs that are locally excited on the metallic ridges of lamellar gratings composed of an array of grooves or slits and illuminated by an incident plane wave. The present approach contrasts with more classical approaches initiated in the 1900s by Fano, Hessel and Oliner (see [24] and references therein) to explain grating anomalies through collective resonance modes of periodic interfaces. Hereafter, we rather study the individual SPPs locally excited on every ridge of the surface. In particular, we focus on the normalized SPP-excitation rate-defined as the ratio between the intensities of the local SPP mode excited on the ridges and of the incident plane wave-as a function of wavelength, incidence angle, grating period, and slit width. It is found that the maximum SPP-excitation rate is very weak, $\approx 10$ for slit arrays, but that it can reach 100 by using resonant grooves. We additionally study how the excitation rate depends on the metal permittivity and show that the dependence is completely different from that of isolated slits or grooves [25]. Our analysis is based on an analytical model that provides closed-form expressions for the SPP-excitation rate and on fully vectorial computation data.

The present article follows a recent letter [26]. It is much more detailed, and additionally provides for the important case of groove arrays that support strong reso- 
nance. It is organized as follows. In Section 2, we define what we call the normalized SPP-excitation rate, and on the basis of the normal-mode orthogonality, we explain how this rate can be calculated by solving Maxwell's equations. In Section 3, we study the influence of the angle of the incident plane wave on the SPP-excitation rate at visible frequencies. It is found that the excitation rate is very low (0.05) in general, except when the illumination angles and wavelengths are close to Rayleigh anomalies, for which one of the spectral orders emerges from the grating at the grazing angle. The computational results are shown to be in quantitative agreement with the predictions of a simplified analytical model that we present in Section 4. As the result of the quantitative agreement, the model allows us to thoroughly analyze and intuitively understand the influence of all the grating parameters on the excitation rate. The influence of the metal conductivity deserves particular attention; it is the purpose of Section 5. Additionally, in Section 6, we use the approximate model to investigate other geometries, such as metallic groove arrays. The geometries that offer very large normalized excitation rates are important in practice, especially for sensor applications. The conclusion is drawn in Section 7.

\section{DEFINITION OF THE NORMALIZED SPP-EXCITATION RATE FOR PERIODIC METALLIC INTERFACE}

We start by considering a gold interface perforated with a periodic array of semi-infinite slits; see Fig. 1(a). The grating period is denoted by $a, w$ represents the slit width, and $f=w / a$ is the slit fill factor. The frequencydependent permittivity $\varepsilon_{\mathrm{m}}$ of gold is taken from [27]. The grating is embedded in air with the relative permittivity

(a)

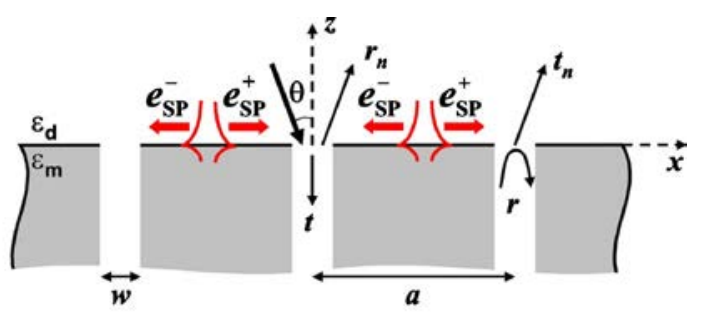

(b)

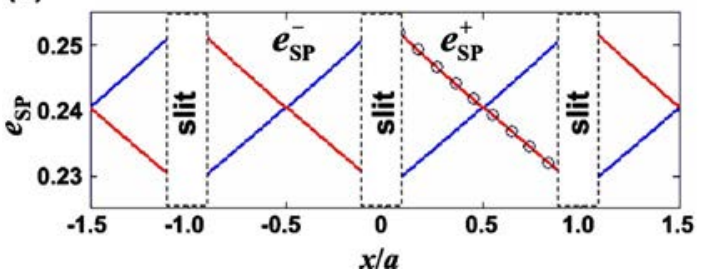

Fig. 1. (Color online) Local excitation of SPPs on the individual ridges of periodic metallic surfaces. (a) Example of an interface between a homogeneous medium (permittivity $\varepsilon_{\mathrm{d}}$ ) and a periodic medium composed of an array of semi-infinite slits. $r_{\mathrm{n}}, t_{\mathrm{n}}, r$, and $t$ are scattering coefficients used in the analytical model of Section 3. $e_{\mathrm{SP}}{ }^{+}$and $e_{\mathrm{SP}}{ }^{-}$represent the normalized excitation rate of SPPs propagating along positive and negative $x$ directions. (b) An example of SPP-excitation rates $\left[e_{\mathrm{SP}}{ }^{+}\right.$: lighter (red online) curve, and $e_{\mathrm{SP}}{ }^{-}$: darker (blue online) curve] calculated for gold $\left(\varepsilon_{\mathrm{m}}\right.$ $=-9.04+1.06 \mathrm{i}), \quad a=0.7 \mu \mathrm{m}, \quad w=100 \mathrm{~nm}, \quad f=w / a=0.143, \quad \lambda$ $=637 \mathrm{~nm}$ and $\theta=0$. The black circles show the SPP damping on flat metal surfaces, $\exp \left(-2 \operatorname{Im}\left(k_{\mathrm{SP}}\right) x\right)$. $\varepsilon_{\mathrm{d}}=\left(n_{\mathrm{d}}\right)^{2}=1$ and is assumed to be illuminated by a TMpolarized plane wave (magnetic field parallel to the interface), since SPPs are not excited for the other polarization. The incident wavelength is denoted by $\lambda$ and the incidence angle by $\theta$. The scattered field can be calculated with fully vectorial computational tools. We use here a modal method [28] that is derived from the rigorous coupled wave analysis (RCWA) [29], and which additionally allows us to satisfy outgoing wave conditions in periodic media (the semi-infinite slit array). The approach holds for an illumination by either a plane wave of the air cladding or by a Bloch mode of the slit array. Hereafter, we denote by $\left[H_{\mathrm{y}}(\mathrm{x}, \mathrm{z}), E_{\mathrm{x}}(\mathrm{x}, \mathrm{z}), E_{\mathrm{z}}(\mathrm{x}, \mathrm{z})\right]$ the total field scattered by the perforated interface. Indeed, SPPs that propagate in opposite directions are locally excited on the flat surfaces (the ridges) between the slits.

To calculate the SPP excitation, we refer to a normalmode formalism recently developed for analyzing SPPs that are launched by sub- $\lambda$ slits on metallic surfaces $[21,25,30]$ and other isolated indentations [31]. Hereafter, we assume that the SPP modes are normalized such that their magnetic fields are unitary on the interface at $x=z=0$. Thus the transverse field components of the forward-propagating SPP may be written as $\left[H_{\mathrm{y}}, E_{\mathrm{z}}\right]=\left[H_{\mathrm{SP}}(z), E_{\mathrm{SP}}(z)\right] \exp \left(\mathrm{j}_{\mathrm{SP}} x\right)=\left[1, k_{\mathrm{SP}} /\left(\kappa \omega \varepsilon_{0}\right)\right]$ $\times \exp \left(\mathrm{j} \gamma_{\mathrm{SP}} z\right) \exp \left(\mathrm{j} k_{\mathrm{SP}} x\right)$, with $\kappa=\varepsilon_{\mathrm{d}}$ or $\varepsilon_{\mathrm{m}}$ for $z>0$ or $z<0$, $\gamma_{\mathrm{SP}}=\left(\varepsilon_{\mathrm{d}} k_{0}{ }^{2}-k_{\mathrm{SP}}{ }^{2}\right)^{1 / 2}$ in air and $\left(\varepsilon_{\mathrm{m}} k_{0}{ }^{2}-k_{\mathrm{SP}}{ }^{2}\right)^{1 / 2}$ in metal. $k_{\mathrm{SP}}=k_{0}\left[\varepsilon_{\mathrm{d}} \varepsilon_{\mathrm{m}} /\left(\varepsilon_{\mathrm{d}}+\varepsilon_{\mathrm{m}}\right)\right]^{1 / 2}$ denotes the SPP propagation constant along the $x$ axis, and $k_{0}=2 \pi / \lambda=\omega / \mathrm{c}$ is the wave vector modulus in vacuum.

According to the completeness theorem for normal modes of waveguides [32], the total field scattered by the periodic surface can be locally decomposed in every flat section $w / 2+n a<x<-w / 2+(n+1) a$ ( $n$ being a relative integer) as a combination of forward and backward bounded and radiative modes of the planar interface:

$$
\begin{aligned}
& H_{\mathrm{y}}(x, z)=\left[\beta_{\mathrm{SP}}{ }^{+}(x)+\beta_{\mathrm{SP}}{ }^{-}(x)\right] H_{\mathrm{SP}}(z)+\Sigma_{\sigma} a_{\sigma} H_{\mathrm{y}}^{(\sigma)}(x, z), \\
& E_{\mathrm{z}}(x, z)=\left[\beta_{\mathrm{SP}}{ }^{+}(x)-\beta_{\mathrm{SP}}{ }^{-}(x)\right] E_{\mathrm{SP}}(z)+\Sigma_{\sigma} a_{\sigma} E_{\mathrm{z}}^{(\sigma)}(x, z),
\end{aligned}
$$

where the sign $\Sigma$ refers to a summation over a continuum of radiation modes. $\beta_{\mathrm{SP}}{ }^{+}(x)$ and $\beta_{\mathrm{SP}}{ }^{-}(x)$ are the magnetic amplitudes of the SPPs propagating in positive and negative $x$ directions, respectively. Like the total field itself, they are pseudo-periodic functions of the $x$ coordinate, $\beta_{\mathrm{SP}}{ }^{+}(x+a)=\exp \left[\mathrm{j} n_{\mathrm{d}} k_{0} a \sin (\theta)\right] \beta_{\mathrm{SP}}{ }^{+}(x)$. Using the orthogonality between bounded and radiative modes $\int\left[E_{z}{ }^{(\sigma)}\right.$ $\left.\times(x, z) H_{\mathrm{SP}}(z)-E_{\mathrm{SP}}(z) H_{y}{ }^{(\sigma)}(x, z)\right] \mathrm{d} z=0$ [21], we obtain the normalized excitation rates for SPPs propagating in either the positive or negative $x$ directions,

$$
\begin{aligned}
e_{\mathrm{SP}}{ }^{+}(x)= & \left|\beta_{\mathrm{SP}}{ }^{+}(x)\right|^{2}=\left(4 N_{\mathrm{SP}}\right)^{-2} \mid \int\left[E_{\mathrm{SP}}(z) H_{\mathrm{y}}(x, z)\right. \\
& \left.+E_{\mathrm{z}}(x, z) H_{\mathrm{SP}}(z)\right]\left.\mathrm{d} z\right|^{2},
\end{aligned}
$$




$$
\begin{aligned}
e_{\mathrm{SP}}{ }^{-}(x)= & \left|\beta_{\mathrm{SP}^{-}}(x)\right|^{2}=\left(4 N_{\mathrm{SP}}\right)^{-2} \mid \int\left[E_{\mathrm{SP}}(z) H_{\mathrm{y}}(x, z)\right. \\
& \left.-E_{\mathrm{z}}(x, z) H_{\mathrm{SP}}(z)\right]\left.\mathrm{d} z\right|^{2},
\end{aligned}
$$

where $N_{\mathrm{SP}}=1 / 2 \int E_{\mathrm{SP}}(z) H_{\mathrm{SP}}(z) \mathrm{d} z$ is a normalization constant, approximately given by $N_{\mathrm{SP}} \approx\left|\varepsilon_{\mathrm{m}}\right|^{1 / 2}\left(4 \omega \varepsilon_{0} \varepsilon_{\mathrm{d}}{ }^{3 / 2}\right)$ for $\left|\varepsilon_{\mathrm{m}}\right| \gg \varepsilon_{\mathrm{d}}$.

For the following calculations, we assume that the magnetic field of the incident plane wave is unitary at $x$ $=z=0$. Since our normalized SPP mode also manifests a unitary amplitude at $x=z=0, e_{\mathrm{SP}}{ }^{+}$and $e_{\mathrm{SP}}{ }^{-}$can be viewed as normalized excitation rates. If $e_{\mathrm{SP}}{ }^{ \pm}>1$ (resp. $e_{\mathrm{SP}}{ }^{ \pm}<1$ ), the SPP intensity of the launched SPPs is larger (resp. smaller) than that of the incident illumination; this corresponds to a field enhancement (resp. retrenchment).

Figure 1(b) shows a numerical example. The calculation is performed for $a=0.7 \mu \mathrm{m}$ and $\lambda / a=0.91$. The lighter (red online) and darker (blue online) curves represent the $e_{\mathrm{SP}}{ }^{+}(x)$ and $e_{\mathrm{SP}}{ }^{-}(x)$ functions, respectively, calculated with Eqs. (2a) and (2b). For normal incidence $\left(\theta=0^{\circ}\right)$, they are periodic $e_{\mathrm{SP}}{ }^{+}(x)=e_{\mathrm{SP}}{ }^{+}(x+a)$ and, for symmetry reasons, $e_{\mathrm{SP}}{ }^{+}(x)=e_{\mathrm{SP}}{ }^{-}(-x)$. Within the slit intervals $n a-w / 2<x$ $<n a+w / 2$, the integrals defined by Eqs. (2a) and (2b) may also be calculated, but they are meaningless since there are no air-metal interfaces in those intervals. For every ridge, $e_{\mathrm{SP}}{ }^{+}$exponentially depends on the coordinate $x$ axis. The exponential decay exactly coincides with the SPP damping $\left[\exp \left(-2 \operatorname{Im}\left(k_{\mathrm{SP}}\right) x\right)\right]$ on metallic flat surfaces, which is shown with black circles for one of the ridges. The coincidence is not fortuitous and evidences the soundness of the mode decomposition formalism for quantifying the SPP-excitation rate on the individual ridges [21].

Let us further consider the SPP mode propagating in the positive $x$ direction. We note that $e_{\mathrm{SP}}{ }^{+}$is always larger on the right side of the slit than on the left side (it is the reverse situation for $e_{\mathrm{SP}}{ }^{-}$). The jump is due to several elementary scattering events-including elastic ones with SPPs that are impinging on the individual slits and inelastic ones with other fields (such as the incident plane wave or the quasi-cylindrical waves [8] that scatter SPPs) - that illuminate the individual slits. Note that the net jump exactly compensates the decrease due to SPP damping across every individual ridge. This property that is a direct consequence of the periodicity is general; it does not depend on the geometry (slit width, groove depth, etc.), it holds for both $e_{\mathrm{SP}}{ }^{+}(x)$ and $e_{\mathrm{SP}}{ }^{-}(x)$, and it holds for arbitrary illumination either with plane waves impinging at oblique incidence or with the Bloch modes of the slit array.

Although the present work is devoted to periodic structures, the normalized SPP-excitation rate can also be defined for structures that are not periodic. Figure 2 shows the example of a structure that is composed of eleven grooves in a gold film. For an illumination at $\lambda=800 \mathrm{~nm}$ by an incident Gaussian beam with a $5 \lambda$ waist, the groove depths and widths have been optimized with a simplex method [33] to maximize the energies of the SPPs launched on both sides of the array. For the optimal parameters, the SPP efficiency is as large as 78\%, implying

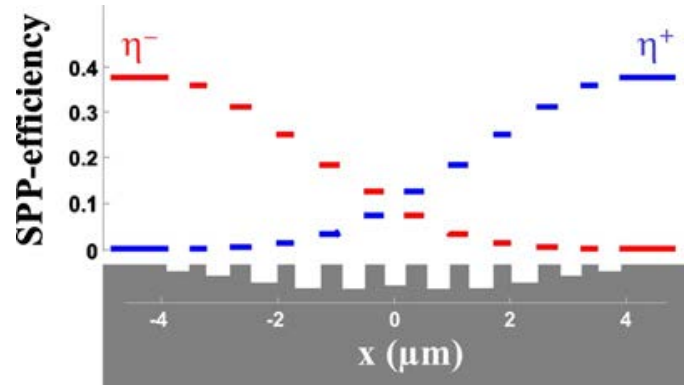

Fig. 2. (Color online) Efficiencies of SPPs locally excited on the ridges of an 11-groove SPP-coupler in a gold substrate illuminated by a normally incident Gaussian beam. The efficiencies are normalized by the power of the Gaussian beam. The optimization is performed for $\lambda=800 \mathrm{~nm}\left(n_{\mathrm{m}}=0.18+5.13 \mathrm{i}\right)$. The coupler is symmetric, and starting from the center $(x=0)$, the width $w$, height $h$, and central location of the five outer grooves are $w=0.32,0.40$, $0.42,0.40,0.41 \mu \mathrm{m} ; h=0.07,0.07,0.07,0.05,0.015 \mu \mathrm{m}$; and $x$ $=0.35,1.12,1.84,2.63,3.38 \mu \mathrm{m}$. The width and depth of the central groove are $w=0.37 \mu \mathrm{m}$ and $\mathrm{h}=0.08 \mu \mathrm{m}$, respectively.

that more than $3 / 4$ of the incident photons are converted into two symmetric SPPs launched in opposite directions. The curves show the normalized SPP-excitation efficiencies $\eta^{+}(x)$ and $\eta^{-}(x)$ that represent the local SPP energy flow propagating to the right or left on every flat interface between the grooves (we use here a slightly different normalization for convenience). It is remarkable to observe that the optimal geometry found by maximizing the SPPs launched on both sides of the array produces a progressive increase of the local efficiency inside the array. As we know from optimizations performed for other beam waists and groove numbers, the present solution contrasts with others (not shown) that present much more abrupt variations of the efficiency and that are also much less robust to fabrication errors.

\section{NORMALIZED SPP-EXCITATION RATES AT VISIBLE FREQUENCIES}

Because the SPP damping over a single ridge is very weak in general [it is only $8 \%$ at $\lambda=633 \mathrm{~nm}$ for gold in Fig. 1(b)], we will not consider the $x$ dependence of $e_{\mathrm{SP}}{ }^{+}(x)$ and $e_{\mathrm{SP}}{ }^{-}(x)$ in the following. Thus the normalized SPPexcitation rate will be considered as the averaged value obtained in the center of every ridge. The rate will be simply denoted by $e_{\mathrm{SP}}{ }^{ \pm}$, with $e_{\mathrm{SP}}{ }^{ \pm}=e_{\mathrm{SP}}{ }^{ \pm}(a / 2)$. We have repeated the calculations of Fig. 1(b) for various values of the angle of incidence $\theta$ and of the illumination wavelength $\lambda$. Figure $3(\mathrm{a})$ shows $e_{\mathrm{SP}}{ }^{+}+e_{\mathrm{SP}}{ }^{-}$in the $\left(\omega-k_{\|}\right)$diagram. The results were obtained with the RCWA for $a$ $=0.7 \mu \mathrm{m}, w=100 \mathrm{~nm}$, and for the gold geometry of Fig. 1(a).

In general, the normalized SPP-excitation rate is very weak on average $\left(\left\langle e_{\mathrm{SP}}{ }^{+}\right\rangle=\left\langle e_{\mathrm{SP}}{ }^{-}\right\rangle \approx 0.05\right)$; it is larger than unity only for some narrow bands in the vicinity of the Rayleigh wavelengths. This will be interpreted in Section 5 as a coherent interference between the individual SPPS and quasi-cylindrical waves $(\mathrm{CWs})$ that are excited and scattered by every slit of the array. Note however that the excitation enhancement is not overwhelmingly strong; the averaged value in the narrow bands is only 4 , a value obtained for the field intensity on a flat metallic interface il- 


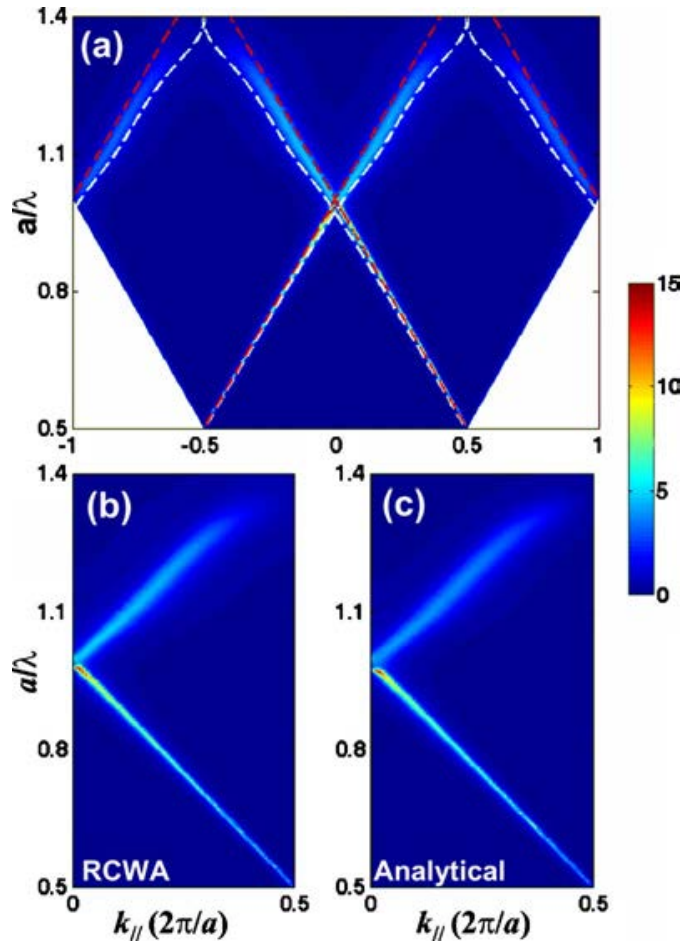

Fig. 3. (Color online) Normalized SPP-excitation rate in the $\left(\omega-k_{\|}\right)$diagram at visible frequencies. (a) Fully vectorial [Eqs. (2a) and (2b)] result showing $e_{\mathrm{SP}}{ }^{+}+e_{\mathrm{SP}}{ }^{-}$as a function of the parallel wave vector $k_{\|}=k_{0} n_{\mathrm{d}} \sin (\theta)$ and the normalized frequency $a / \lambda$. The results hold for the gold geometry of Fig. 1(a) and for $a$ $=0.7 \mu \mathrm{m}$. The dimmer (red online) and brighter (blue online) dashed curves represent the Rayleigh anomalies and the folded dispersion relation of SPPs on flat metal surfaces. (b) and (c) are enlarged views of (a) obtained, respectively, with the RCWA and the approximate model [Eqs. (5a) and (5b)]. Quantitative agreement is achieved.

luminated by a plane wave with a unit amplitude. Indeed, the perforated interface is not a resonant geometry, because every slit drains off an important fraction of the incoming energy carried by the incident SPPs $[9,13]$. The enhancement resonance is more acute in the vicinity of the $\Gamma$ point $\left(e_{\mathrm{SP}}{ }^{+}=e_{\mathrm{SP}}{ }^{-} \approx 15\right)$, where counter propagating SPPs with opposite amplitudes may be resonantly excited with a slightly oblique illumination by breaking the vertical symmetry achieved under normal incidence [34]. The bright bands in Fig. 3(a) generally follow two sets of curves: the Rayleigh wavelengths shown with the dimmer dashed (red online) curves and defined by $k_{\|}+2 n \pi / a$ $= \pm k_{0}$, and the folded dispersion relations of SPPs on flat metallic surfaces $\left(k_{\|}+2 n \pi / a= \pm k_{\mathrm{SP}}\right)$, shown with the brighter dashed (white online) curves. Note that the two sets of curves almost merge at small energies, for which $k_{\mathrm{SP}} \approx k_{0}$. This is because the SPPs exhibit a long tail in the dielectric cladding at small energies and thus are transmitted through slits with an almost unitary transmission coefficient, rendering their dispersion curves close to those of SPPs on flat metallic surfaces.

\section{ANALYTICAL MODEL}

In order to obtain an insight into the influence of the various grating parameters on the normalized SPP-excitation rate, we further propose an analytical model based on classical approximations used for analyzing gratings with sub- $\lambda$ apertures [35-37]. Within the scope of the model, the field above the grating is expressed with a Rayleigh expansion, $H_{\mathrm{y}}(x, z)=\exp \left(\mathrm{j} \alpha_{0} \mathrm{x}\right) \exp \left(-\mathrm{j} \gamma_{0} k_{0} z\right)$ $+\Sigma_{\mathrm{n}} r_{\mathrm{n}} \exp \left(\mathrm{j} \alpha_{\mathrm{n}} x\right) \exp \left(\mathrm{j} \gamma_{\mathrm{n}} k_{\mathrm{n}} \mathrm{z}\right)$, where $r_{\mathrm{n}}$ represents the unknown reflectivity coefficient of the $n$th order $\alpha_{\mathrm{n}}=k_{\|}$ $+2 n \pi / a$, and $\gamma_{\mathrm{n}}{ }^{2} k_{0}{ }^{2}+\alpha_{\mathrm{n}}{ }^{2}=\varepsilon_{\mathrm{d}} k_{0}{ }^{2}$. The electric fields can be obtained from Maxwell's equations, $E_{\mathrm{x}}(x, z)$ $=-\left(\mathrm{j} k_{0}\right)^{-1} \partial H_{\mathrm{y}}(x, z) / \partial z, \quad$ and $\quad E_{\mathrm{z}}(x, z)=\left(\mathrm{j} k_{0}\right)^{-1} \partial H_{\mathrm{y}}(x, z) / \partial x$. The electromagnetic field in the slits is assumed to be composed only of the fundamental propagative Bloch mode of the array. Details of the single-mode approximation can be found in [10]. For the sake of accuracy, we use surface impedance boundary conditions [36,37] rather than the perfect conductor approximation [38] for matching the boundary conditions at the interface $z=0$. According to Eqs. (2a) and (2b), the excitation rates are given by

$$
\begin{aligned}
e_{\mathrm{SP}}^{+}(x)= & \Sigma_{\mathrm{n} \neq 0}\left|r_{\mathrm{n}}\right|^{2}\left(4\left|N_{\mathrm{SP}}\right|\right)^{-2} \mid \int\left[E_{\mathrm{SP}}(z) H_{\mathrm{y}}^{(\mathrm{n})}(x, z)+E_{\mathrm{z}}^{(\mathrm{n})}\right. \\
& \left.\times(x, z) H_{\mathrm{SP}}(z)\right]\left.\mathrm{d} z\right|^{2}, \\
e_{\mathrm{SP}}{ }^{-}(x)= & \Sigma_{\mathrm{n} \neq 0}\left|r_{\mathrm{n}}\right|^{2}\left(4\left|N_{\mathrm{SP}}\right|\right)^{-2} \mid \int\left[E_{\mathrm{SP}}(z) H_{\mathrm{y}}^{(\mathrm{n})}(x, z)-E_{\mathrm{z}}^{(\mathrm{n})}\right. \\
& \left.\times(x, z) H_{\mathrm{SP}}(z)\right]\left.\mathrm{d} z\right|^{2},
\end{aligned}
$$

where

$$
r_{\mathrm{n}}=\delta_{\mathrm{n}, 0} \frac{\gamma_{\mathrm{n}}-Z_{\mathrm{s}}}{\gamma_{\mathrm{n}}+Z_{\mathrm{s}}}+\frac{2\left(Z_{\mathrm{s}}-n_{\mathrm{eff}}\right) f_{0} g_{\mathrm{n}} \gamma_{0}}{\left(\gamma_{0}+Z_{\mathrm{s}}\right)\left(\gamma_{\mathrm{n}}+Z_{\mathrm{s}}\right)(1-I)}
$$

In Eqs. (3) and (4), $\delta_{\mathrm{n}, 0}=1$ if $n=0$ and 0 otherwise, $n_{\text {eff }}$ denotes the effective index of the fundamental slit mode $\left(n_{\mathrm{eff}} \approx 1\right), Z_{\mathrm{s}}=\varepsilon_{\mathrm{m}}{ }^{-1 / 2}$ is the metal surface impedance supposed to be independent of $k_{\|}$for a fixed wavelength [36], $I=\Sigma_{\mathrm{n}}\left[\left(Z_{\mathrm{s}}-n_{\text {eff }}\right) f g_{\mathrm{n}}^{2}\right] /\left(\gamma_{\mathrm{n}}+Z_{\mathrm{s}}\right)$ with $n$ running over all integers, $g_{\mathrm{n}}=1 / w \int_{-w / 2}^{w / 2} \varphi(x) \exp \left(j \alpha_{\mathrm{n}} x\right) \mathrm{d} x, \quad H_{\mathrm{y}}{ }^{(\mathrm{n})}(x, z)$ $=\exp \left(\mathrm{j} \alpha_{\mathrm{n}} x\right) \exp \left(\mathrm{j} \gamma_{\mathrm{n}} k_{0} z\right)$, and $E_{\mathrm{z}}{ }^{(\mathrm{n})}(x, z)=\left(\mathrm{j} k_{0}\right)^{-1} \partial H_{\mathrm{y}}{ }^{(\mathrm{n})} / \partial x$. The fundamental slit mode profile $\varphi(x)$ can be figured out analytically by using the surface impedance boundary conditions [37] and is normalized such that $1 / w \int_{-w / 2}^{w / 2}|\varphi(x)|^{2} \mathrm{~d} x=1$. Note that the specularly reflected plane wave does not contribute to the excitation of SPPs and has been removed from the plane wave expansion of Eqs. (3a) and (3b). The latter shows that $e_{\mathrm{SP}}{ }^{ \pm}$stems from a summation of the contributions from all the gratingreflection orders. We have analytically shown that, in the sum, only a single reflection order significantly contribute to $e_{\mathrm{SP}}{ }^{ \pm}$. This result, which is consistent with one's classical idea that SPPs are launched on periodic metallic interfaces when their momentum matches that of the incident illumination through a multiple of the grating momentum, can be used to drastically simplify the expressions in Eqs. (3a) and (3b). Hereafter we will be concerned only by the SPPs that are launched through firstorder scattering, so that the main contribution in Eqs. (3a) and (3b) is due to $n=+1$ or -1 . By further neglecting 
the field penetration in the metal, the SPP-excitation rate resulting from a first-order scattering of the incident plane wave may be written

$$
\begin{aligned}
& e_{\mathrm{SP}}{ }^{+}=R_{1} Q_{1}=\left|r_{1}\right|^{2}\left|\frac{\gamma_{\mathrm{SP}}\left(\alpha_{1}+k_{\mathrm{SP}}\right)}{k_{\mathrm{SP}}\left(\gamma_{1} k_{0}+\gamma_{\mathrm{SP}}\right)}\right|^{2}, \\
& e_{\mathrm{SP}}{ }^{-}=R_{-1} Q_{-1}=\left|r_{-1}\right|^{2}\left|\frac{\gamma_{\mathrm{SP}}\left(\alpha_{-1}-k_{\mathrm{SP}}\right)}{k_{\mathrm{SP}}\left(\gamma_{-1} k_{0}+\gamma_{\mathrm{SP}}\right)}\right|^{2},
\end{aligned}
$$

where $R_{ \pm 1}=\left|r_{ \pm 1}\right|^{2} . Q_{1}$ and $Q_{-1}$ refer to the fraction factor in the rightmost side of Eqs. (5a) and (5b). They essentially come from the overlap integral of the SPP fields and of the positive and negative first-order plane waves. In the vicinity of the Rayleigh wavelengths $\lambda_{\mathrm{R}}=a\left(1 \pm k_{\|} / k_{0}\right)$, where the normalized excitation rate is maximum, $Q_{ \pm 1}$ take the simplified form

$$
Q_{ \pm 1} \approx 4\left|1+\left[2 \epsilon_{\mathrm{m}}\left(\lambda^{ \pm 1}-\lambda\right) / a\right]^{1 / 2}\right|^{-2}
$$

and peak at $\lambda=\lambda^{ \pm 1}$, for which $e_{\mathrm{SP}}{ }^{ \pm} \approx 4 R_{ \pm 1}\left(\lambda^{( \pm 1)}\right)$. Figures 3(b) and 3(c) compare the analytical formula of Eqs. (5a) and (5b), with fully vectorial computational results obtained with the RCWA. For the sake of better comparison, in Fig. 4 we show two spectra of the excitation rate obtained for $\theta=10^{\circ}$ [Fig. 4(a)] and $\theta=0^{\circ}$ [Fig. 4(b)]. The excitation-rate maxima in Fig. 4(a) are located in the vicinity of the Raleigh wavelengths $\lambda_{\mathrm{R}} / a=1 \pm \sin (\theta)$ indicated by the lighter (red online) vertical dashed lines. For normal incidence, $e_{\mathrm{SP}}{ }^{+}$and $e_{\mathrm{SP}}{ }^{-}$are equal, and their maxima are obtained exactly at the Rayleigh wavelengths [see Fig. 4(b)]. As in Fig. 3(b), the normalized SPPexcitation rate predicted with the approximate model [darker (blue online) curves] quantitatively agree with the RCWA data [lighter (red online) curves].

Our current understanding of the SPP-excitation on dressed metallic interfaces is based on SPP multiple scattering. Intuitively, one expects that, under light illumination, every individual slit launches SPPs in the positive and negative directions, and that the SPPs further elastically scatter on the nearby slits, with possibly multiple scatterings involving back-reflection. A model (called pure SPP model hereafter, to emphasize that only SPPs are assumed to transport the electromagnetic information on the surface) that allows to us calculate the SPP-excitation rates within this fully elastic framework has been recently developed to indentify the role of SPPs in extraordinary optical transmission. The SPP-coupled-mode equations are readily obtained for periodic structures like the slit array considered in the present work, and details can be found in the Supplementary Section of [9]. The pure SPP model predictions are shown with the dotted-dashed curves in Fig. 4(a) and 4(b). They are rather accurate; for instance, the model quantitatively predicts the very weak values of the excitation rate far away from $\lambda_{R}$ and the "exact" location of the maxima at a wavelength close to $\lambda_{R}$. However, it underestimates the maximum excitation rate and does not predict the sharp peak obtained for $\theta=0^{\circ}$ or for $e_{\mathrm{SP}}{ }^{-}$at $\theta=10^{\circ}$. Indeed the intuitive picture, which assumes that the electromagnetic interaction between the slits is solely mediated by SPP fields, is incomplete, and other waves different from the SPPs, the (CWs) [7,21,39],

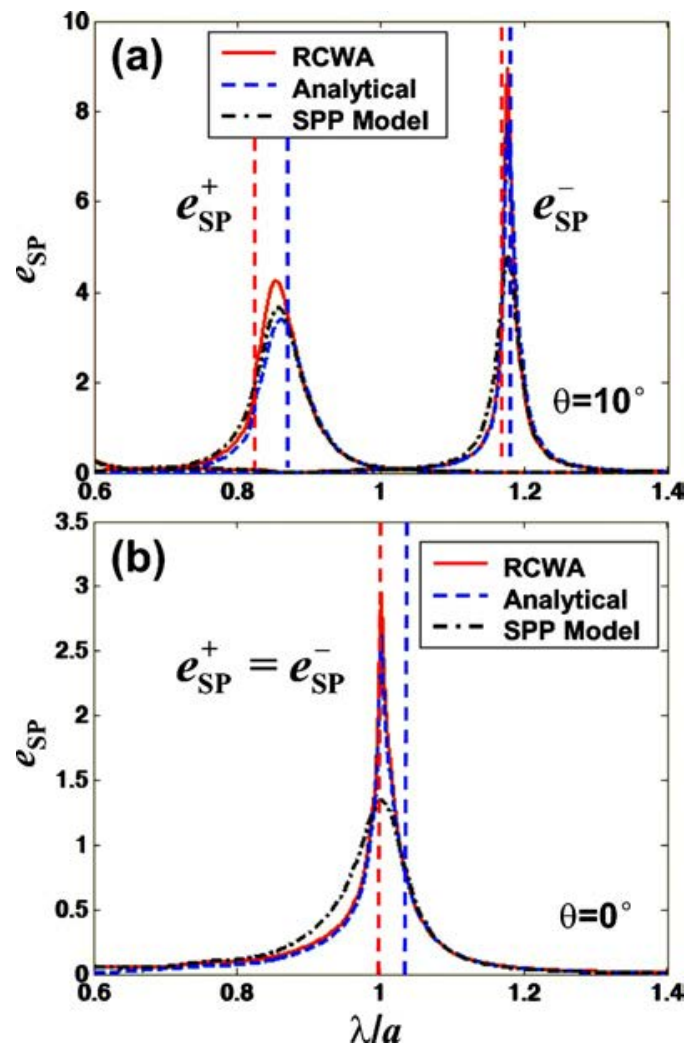

Fig. 4. (Color online) Normalized SPP-excitation rate spectrum for fixed angles of incidence. (a) $\theta=10^{\circ}$ and (b) $\theta=0^{\circ}$. Solid (red online), dashed (blue online), and dotted-dashed (black online) curves refer to the data obtained with the RCWA, the approximate model, and the pure SPP model, respectively. Note that $e_{\mathrm{SP}}{ }^{+}$ and $e_{\mathrm{SP}}{ }^{-}$merge for normal incidence because of the symmetry. Other parameters used for the computation are $a=0.7 \mu \mathrm{m}$ and $w=0.143 a$. The vertical dashed lines (red online) represent the Raleigh wavelengths, $\lambda_{\mathrm{R}} / a=1 \pm \sin (\theta)$. The vertical dashed lines (blue online) represent the SPP wavelengths, $\lambda_{\mathrm{SP}} / a=\left[\varepsilon_{\mathrm{m}} \varepsilon_{\mathrm{d}} /\left(\varepsilon_{\mathrm{m}}\right.\right.$ $\left.\left.+\varepsilon_{\mathrm{d}}\right)\right]^{1 / 2} \pm \sin (\theta)$.

are also launched on the ridges surfaces. Like the SPPs, these waves further scatter on the nearby slits, and are converted into SPPs [8]. Since the sole approximation of the pure SPP model consists in neglecting the additional $\mathrm{CW}$, one deduces that the appearance of sharp peaks for the excitation rate is due to multiple CW-to-SPP crossconversions that occur at every individual slit and that constructively interfere for $\lambda \approx \lambda_{R}$.

As evidenced by Figs. 3 and 4, the agreement between the analytical model predictions and the fully vectorial RCWA data is quantitative, and the model can be used with confidence to analyze the impact of various grating parameters on the normalized SPP-excitation rate. This is the purpose of Section 5 .

\section{PROPERTIES OF SPP-EXCITATION RATE}

In this section, we again consider the slit array scattering problem of Fig. 1(a), and study the influence of various geometrical and material parameters on the normalized SPP-excitation rate. In particular we analyze the influence of the metal conductivity and of the slit fill factor. 


\section{A. Influence of Metal Conductivity}

It is important to understand how the SPP-excitation rate scales as the metal permittivity increases. By using the analytical model, we have calculated $e_{\mathrm{SP}}{ }^{+}$and $e_{\mathrm{SP}}{ }^{-}$by varying the incident wavelength from the visible to the near-infrared. The results are shown in Fig. 5 for $\theta=10^{\circ}$ and $0^{\circ}$. The spectra are obtained by varying the wavelength for several values of the grating period from $a$ $=0.7$ to $3.1 \mu \mathrm{m}$, by scaling all the grating dimensions, and by using the gold permittivity dependence tabulated in [27]. Two general trends that are valid for oblique [Fig. 5(a)] or normal [Fig. 5(b)] incidence are observed. First, the width $\Delta \lambda$ of the SPP-excitation branches narrows as the metal conductivity increases. From Eq. (6), we have analytically shown that the peak width $\Delta \lambda$ is predominantly due to the special dependence of $Q_{ \pm 1}$. It is easily found that the normalized peak width $\Delta \lambda / a$ of $Q_{1}$ or $Q_{-1}$ is equal to $(2-\sqrt{ } 2)\left|\varepsilon_{\mathrm{m}}\right|^{-1}$ at normal incidence, showing $\left|\varepsilon_{\mathrm{m}}\right|^{-1}$ scaling as the metal permittivity increases at long wavelengths. This trend will be interpreted below as the consequence of an increased SPP propagation length. Second, except at visible frequencies, the maxima of $e_{\mathrm{SP}}{ }^{+}$and $e_{\mathrm{SP}}{ }^{-}$ (denoted $e_{\mathrm{SP}}{ }^{(\max )}$ hereafter) are found to be almost independent of the metal conductivity, as also shown in Fig. 5(c) that shows the extracted maximum values at normal incidence. This result is unexpected in our opinion. Indeed, for isolated slits $[21,25]$ or subwavelength indentations in general [31], the normalized SPP-excitation rate rapidly drops (it scales as $\left|\varepsilon_{\mathrm{m}}\right|^{-1}$ ) as we move from visible to infrared frequencies. This is easily understood if one considers that the SPP mode spreads farther and farther away in the dielectric half-space as the wavelength in- creases, and that spatially delocalized fields cannot be excited efficiently by subwavelength scatterers. Naïvely, one may anticipate this general trend to be observed with arrays of subwavelength indentations. Actually the grating case is much more intricate. First, restructuring the discussion to the sole contribution of SPPs (by neglecting again the impact of the $\mathrm{CWs}$ ), we may consider the pure SPP model and analyze the multi-scattering process of SPPs by every individual slit. Within the scope of the model, the peak maximum is approximately given by $|\beta|^{2} /|1-(\tau+\rho)|^{2}$ at normal incidence [9], where $\tau$ and $\rho$ represent the SPP transmittance and reflectance coefficients, and $\beta$ is the SPP-generation coefficient at a single slit [see insets in Fig. 5(d)]. For narrow slits, we approximately have $\tau-1 \approx \rho$ as the first-Born approximation applies; see [8] for details. Thus the peak maximum can be written as $|\beta|^{2} /\left[4|1-\tau|^{2}\right]$. We have calculated the SPP-scattering coefficients with a fully vectorial method [13]. In Fig. 5(d), we plot $|\beta|^{2}$ and $|1-\tau|^{2}$ as a function of the metal conductivity. It is clear that both efficiencies scale as $\left|\varepsilon_{\mathrm{m}}\right|^{-1}$, which is shown with a dashed-dotted curve. As a result, the excitation rate predicted by the pure SPP model remains constant as the metal permittivity varies, as shown by the dashed curve in Fig. 5(c). The physical reason can be qualitatively understood as follows. As the metal conductivity increases, fewer SPPs are launched by the incident plane wave at every individual slit $\left(|\beta|^{2} \propto\left|\varepsilon_{\mathrm{m}}\right|^{-1}\right)$. Meanwhile the SPPs are less scattered by adjacent slits $\left(|1-\tau| \propto\left|\varepsilon_{\mathrm{m}}\right|^{-1 / 2}\right.$ ), implying that the gathering (propagation) length of SPPs on the corrugated metallic surface becomes longer. It should be mentioned that these properties are not specific to slit geometries and are also ob-
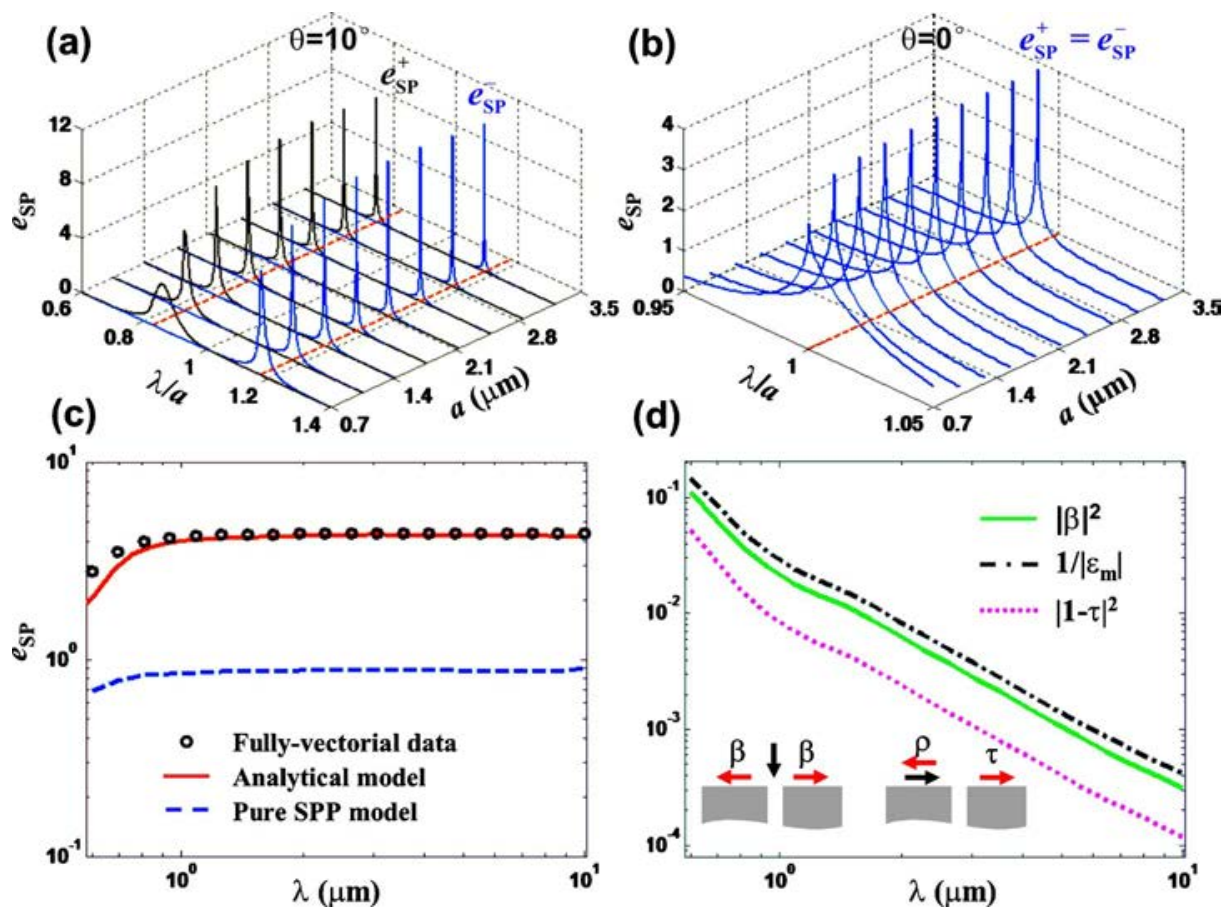

Fig. 5. (Color online) Influence of metal conductivity on the normalized SPP-excitation rate spectrum. (a) and (b) Spectra are calculated as a function of the wavelength of the incident illumination for various grating periods, so that the front and rear curves correspond to visible and infra red frequencies, respectively. (a) $\theta=10^{\circ}$ and (b) $\theta=0^{\circ}$. The dashed (red online) lines under the peaks denote the Rayleigh wavelengths. (c) Maxima of the excitation rate at normal incidence. The circles are fully vectorial data obtained with RCWA. The solid (red online) and dashed (blue online) curves, respectively, refer to the predictions of the analytical and pure SPP models. (d) Dependence of $|\beta|^{2},|1-\tau|^{2}$ on the metal conductivity. The variation of $\left|\varepsilon_{\mathrm{m}}\right|^{-1}$ is also shown as a reference. The insets show the definition of $\beta$, $\tau$, and $\rho$. 
tained for hole chains [9]. If one neglects SPP backreflections, the length can be written as $L_{\mathrm{SP}} \approx$ $-a(2 \ln |\tau|)^{-1}$. Thus as the metal permittivity increases, fewer SPP are launched by every individual slit, but the weaker coupling is exactly compensated by the fact that every slit gathers SPPs that are launched at slits farther and farther away.

Although the pure-SPP model predicts the main special characteristics, it is only qualitative, as evidenced by the curves in Fig. 5(c). Indeed, full assessment requires considering the CWs that are launched by the individual slits-in addition to the SPPs-and their further crossconversion to SPPs through scattering by adjacent slits [8]. This process, which is actually the dominant one even at near-infrared frequencies [Fig. 5(c)], relies on scaling laws that are similar to those previously discussed in the pure SPP model; the cross-conversion efficiency again decreases as the wavelength increases [8], but the decrease is compensated by the increased gathering length over which the cross-conversion processes are gathered at every single aperture. The overall effect is independent of the wavelength, as evidenced by the nearly constant difference between the dashed and solid curves at long wavelengths in Fig. 5(c).

\section{B. Influence of the Slit Width}

Figure 6(a) shows the dependence of the normalized SPPexcitation rate on the slit fill factor $f=w / a$. The computational results obtained with the RCWA hold for normal in-
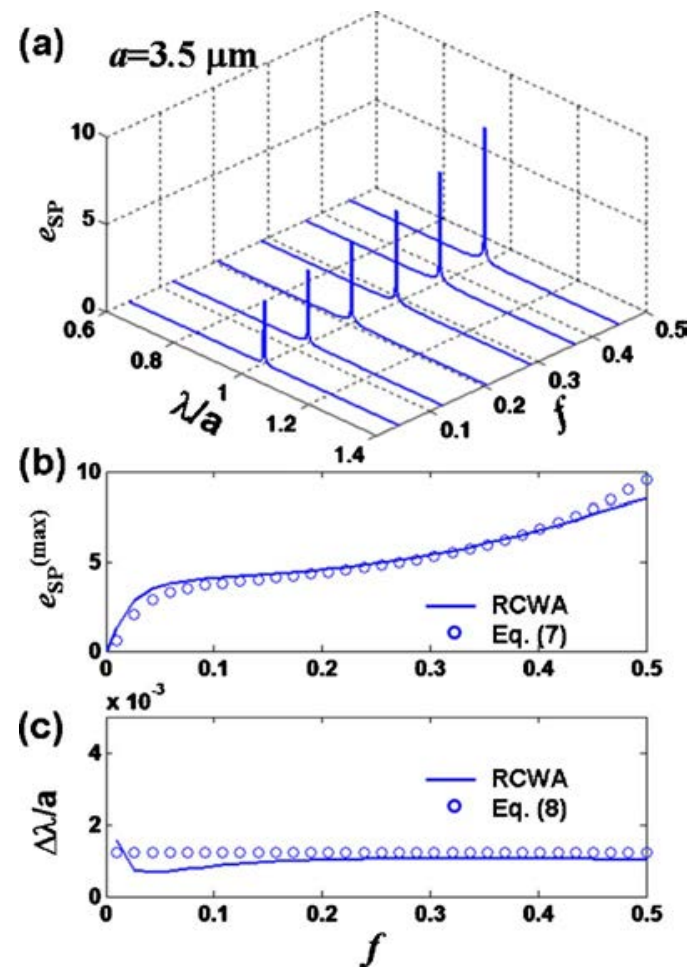

Fig. 6. (Color online) Influence of the slit width. (a) Normalized SPP-excitation rate as a function of the wavelength for $a$ $=3.5 \mu \mathrm{m}$ and for various slit widths $w(f=w / a)$. The results are obtained with the RCWA for normal incidence $\left(e_{\mathrm{SP}}{ }^{+}=e_{\mathrm{SP}}{ }^{-}=e_{\mathrm{SP}}\right)$. (b) Peak value of $e_{\text {SP. }}$ (c) Peak width $\Delta \lambda$. In (b) and (c), the RCWA data and analytical data are shown with solid curves and circles, respectively. cidence and for a grating period $a=3.5 \mu \mathrm{m}$. As discussed in Subsection 5.A, $e_{\mathrm{SP}}$ is always maximum for $\lambda=\lambda_{\mathrm{R}}$ for normal incidence. Figures 6(b) and 6(c) show the variations of the peak excitation rate $e_{\mathrm{SP}}{ }^{(\max )}$ and of the peak width $\Delta \lambda$ with $f$. The width weakly depends on $f$, and only $e_{\mathrm{SP}}{ }^{(\max )}$ exhibits an S-shaped variation for small fill factors. The weak dependence is because the occurrence of the Rayleigh anomaly is independent of the slit width for a fixed grating period. From Eqs. (5) and (6), we have shown analytically that this variation is due to $R_{ \pm 1}\left(Q_{ \pm 1}\right.$ is independent of $f$ ) and is approximately given by

$$
e_{\mathrm{SP}}^{(\max )} \approx\left|4 f n_{\mathrm{m}} \operatorname{sinc}(\pi f) /\left(1+2 f n_{\mathrm{m}} \operatorname{sinc}^{2}(\pi f)\right)\right|^{2},
$$

showing that the peak SPP-excitation rate initially grows quadratically with $f$ for $f<1 /\left|n_{\mathrm{m}}\right|$, where $\mathrm{n}_{\mathrm{m}}=\varepsilon_{\mathrm{m}}{ }^{1 / 2}$. In addition, the normalized peak width is approximately given by

$$
\Delta \lambda / a \approx(2-\sqrt{ } 2)\left|Z_{\mathrm{s}}\right|^{2},
$$

showing that the peak width is independent of the slit width. The analytical model (circles) predictions well match the fully vectorial computational data (solid curves) in Fig. 6(c). For large widths $(f>0.5)$, the singlemode approximation ceases to be valid, and the analytical model cannot be used with confidence.

\section{GROOVED GRATINGS}

So far, we have investigated the SPP-excitation rate on the ridges of gratings consisting of semi-infinite slit arrays. Let us now consider gratings composed of periodic array of grooves, as shown in the inset of Fig. 7(a). Assuming a single-mode approximation in the groove [10], the normalized SPP-excitation rate on the ridges (between the grooves) can be straightforwardly calculated using the approximate model. We obtain

$$
\begin{aligned}
& e_{\mathrm{SP}}{ }^{+}=R_{1} Q_{1}=\left|r_{1}+\frac{t t_{1} r_{\mathrm{b}} u^{2}}{1-u^{2} r_{\mathrm{b}} r}\right|^{2}\left|\frac{\left(\alpha_{1}+k_{\mathrm{SP}}\right) k_{\mathrm{d}}}{\left(\gamma_{1} k_{0}+k_{\mathrm{d}}\right) k_{\mathrm{SP}}}\right|^{2}, \\
& e_{\mathrm{SP}}{ }^{-}=R_{-1} Q_{-1}=\left|r_{-1}+\frac{t t_{-1} r_{\mathrm{b}} u^{2}}{1-u^{2} r_{\mathrm{b}} r}\right|^{2}\left|\frac{\left(\alpha_{-1}-k_{\mathrm{SP}}\right) k_{\mathrm{d}}}{\left(\gamma_{-1} k_{0}+k_{\mathrm{d}}\right) k_{\mathrm{SP}}}\right|^{2},
\end{aligned}
$$

where $u=\exp \left(\mathrm{j} n_{\text {eff }} k_{0} h\right)$ with $h$ being the groove depth; $r_{\mathrm{b}}$ $=\left(n_{\text {eff }}-Z_{\mathrm{s}}\right) /\left(n_{\text {eff }}+Z_{\mathrm{s}}\right)$ is the reflectance coefficient of the fundamental slit mode at the groove-substrate interface; $t=2 g_{0} \gamma_{0} /\left[\left(\gamma_{0}+Z_{\mathrm{s}}\right)(1-I)\right]$ and $r=\left(Z_{\mathrm{s}}-n_{\mathrm{eff}}\right)^{-1}\left[2 n_{\mathrm{eff}} /(1-I)\right.$ $\left.-Z_{\mathrm{s}}-n_{\text {eff }}\right]$ are, respectively, the transmittance and reflectance coefficients of the fundamental slit mode at the groove entrance; and

$$
t_{1}=\frac{f g_{1}\left[Z_{\mathrm{s}}+n_{\mathrm{eff}}+\left(Z_{\mathrm{s}}-n_{\mathrm{eff}}\right) r\right]}{\gamma_{1}+Z_{\mathrm{s}}},
$$



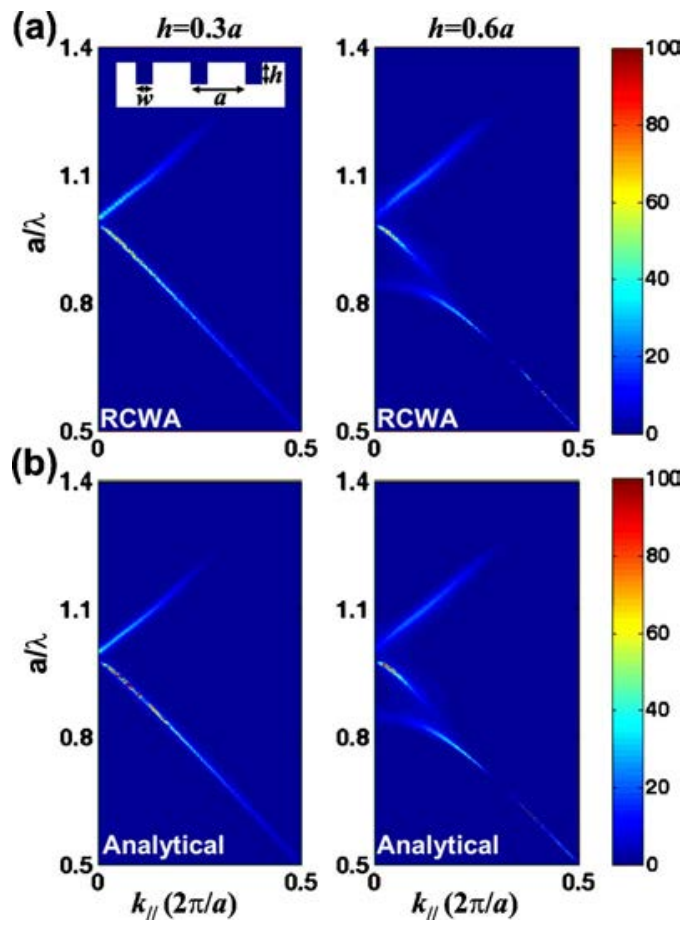

Fig. 7. (Color online) Normalized SPP-excitation rate on the ridges of a grating composed of a periodic array of grooves in gold. (a) RCWA data obtained for groove depths $h=0.3 a$ and $0.6 a$, $a=0.7 \mu \mathrm{m}, w=100 \mathrm{~nm}$. (b) Corresponding results obtained with the approximate model.

$$
t_{-1}=\frac{f g_{-1}\left[Z_{\mathrm{s}}+n_{\mathrm{eff}}+\left(Z_{\mathrm{s}}-n_{\mathrm{eff}}\right) r\right]}{\gamma_{-1}+Z_{\mathrm{s}}},
$$

represent the scattering coefficients of the slit mode into the positive and negative first grating orders. Note that only the \pm 1 orders are considered in the simplified treatment. $R_{ \pm 1}$ is affected not only by the field that is directly reflected from the groove apertures (as for the slit case), but also by the field reflected from the groove bottoms, leading to possible groove resonances. This is evidenced by Fig. 7 , which shows the $\left(\omega-k_{\|}\right)$diagrams of $e_{\mathrm{SP}}{ }^{+}+e_{\mathrm{SP}}{ }^{-}$ for two groove depths $h=0.3 a$ and $0.6 a$. Quantitative agreement is achieved between the fully vectorial RCWA computational data [Fig. 7(a)] and the model predictions of Eq. (9) [Fig. 7(b)].

There are several noticeable differences with the semiinfinite slit geometry. First, the excitation rate may reach much larger values $\left(e_{\mathrm{SP}} \approx 100\right)$ when the grooves resonate. The width $\Delta \lambda$ of each band is also narrower than that obtained for slits. More importantly, as a result of the additional groove resonance condition, new bands far away from the Rayleigh wavelengths are found. This is illustrated by the $\left(\omega-k_{\|}\right)$diagram obtained for $h=0.3 a$, where the lower band splits into two branches for $a / \lambda \approx 0.9$.

Figures 8(a) and 8(b) show the normalized SPPexcitation rate for normal incidence. Again we consider two groove depths, $h=0.3 a$ and $0.6 a$. All the calculations are performed for $a=0.7 \mu \mathrm{m}$ with the RCWA (red-solid curves) or with the approximate model (blue-dashed curves). It is clear that only a single peak appears at the Rayleigh anomaly for $h=0.3 a$. In comparison with Fig. 3(b) obtained for slits, a much stronger excitation rate

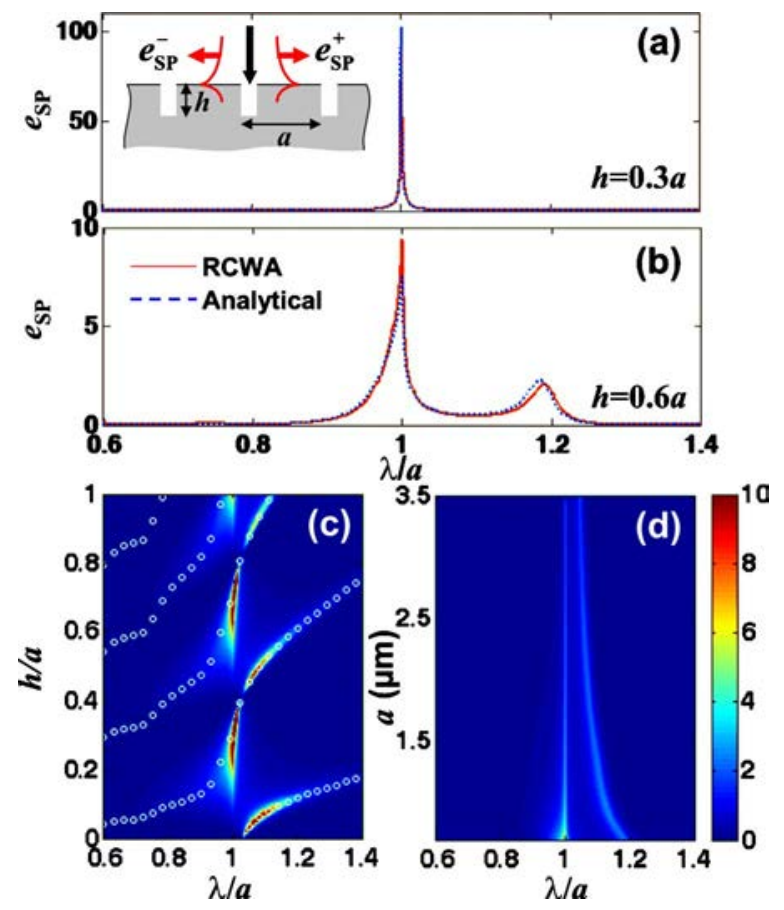

Fig. 8. (Color online) Normalized SPP-excitation rate for periodic groove arrays in gold at normal incidence. (a) and (b): Spectra obtained for $h=0.3 a$ (a) and $h=0.6 a$ (b). The RCWA data and analytical results are denoted by solid (red online) and dotted (blue online) curves, respectively. (c) Excitation rate as a function of the wavelength and of the groove depth for $a=0.7 \mu \mathrm{m}$. The circles indicate the location of the groove resonances according to Eq. (10). (d) Excitation rate as a function of the wavelength (horizontal axis) and of the grating period (vertical axis) for $h=0.6 a$. As for slits, the peak width narrows and the peak value remains constant as the metal permittivity increases.

$(\approx 100)$ is found and the peak width $\Delta \lambda$ is remarkably narrower. Interestingly, another peak appears in the vicinity of the Rayleigh anomaly for $h=0.6 a$. To explain this, we continuously vary the groove depth in Fig. 8(c). It is found that several discrete bands alternately appear at large depths, which relate to the Fabry-Perot resonance in the groove. The resonance condition reads

$$
\begin{aligned}
& 2 \operatorname{Re}\left(n_{\mathrm{eff}}\right) k_{0} h+\arg \left(r_{\mathrm{b}}\right)+\arg (r) \approx 2 \operatorname{Re}\left(n_{\mathrm{eff}}\right) k_{0} h+\arg (r) \\
& \quad=0 \text { (modulo } 2 \pi)
\end{aligned}
$$

since $r_{\mathrm{b}}$ is approximately equal to unity. The bright bands in Fig. 8(c) result from two resonance mechanisms. The first mechanism holds in the vicinity of Rayleigh wavelengths and is exactly the same as that previously discussed for semi-infinite slit arrays. Note that this mechanism is almost systematically assisted by a groove resonance. The reason is that $\arg (r)$ varies rapidly for $\lambda$ $\approx \lambda_{\mathrm{R}}[14]$, which implies that the resonance condition of Eq. (11) is always satisfied when a spectral order emerges from the grating at the grazing angle. The second mechanism is a pure Fabry-Perot resonance that may occur far away from any passing-off of a diffracted order. In Fig. 8(c), we have additionally plotted the $\lambda$ - $h$ curves (white circles) associated with the resonance condition of Eq. (11), which accordingly follow the positions of the bright bands associated with the two identified mechanisms. Figure 8(d) shows the influence of the metal permittivity 
as the grating period is varied from $a=0.7$ to $3.5 \mu \mathrm{m}$. It is apparent that, like the slit case, $e_{\mathrm{SP}}{ }^{(\max )}$ is almost independent of the metal permittivity, and that the peak width $\Delta \lambda$ narrows as $a$ increases. In addition, we observe that the small-energy peak exhibits a blue shift; this is easily understood by considering the dispersion properties of $n_{\text {eff }}$ and $\arg (r)$ leading to the Fabry-Perot resonance in Eq. (11).

\section{CONCLUSION}

In summary, we have theoretically investigated the amount of SPPs that are excited on every individual ridge of lamellar metallic gratings. For gratings consisting of periodic arrays of slits, the normalized SPP-excitation rate is in general rather weak, except when the illumination angle and wavelength are close to the Rayleigh wavelength (Figs. 3 and 4). Additionally, we have shown [Eqs. (5) and (6)] that under normal illumination, the maximum $e_{\mathrm{SP}}{ }^{(\max )}$ of the excitation rate is always met at the Raleigh wavelength $(\lambda=a)$. Perhaps counterintuitively and in contrast with the SPP-excitation rate of isolated indentations, we found that $e_{\mathrm{SP}}{ }^{(\max )}$ is virtually independent of the metal permittivity (Fig. 5). As we scale the grating parameters and move from visible to infrared light, $e_{\mathrm{SP}}{ }^{(\max )}$ remains constant; only the peak width of the normalized SPP-excitation rate is narrowed. These results are not specific to slit arrays, as they also hold for hole arrays. We have additionally shown that, because of Fabry-Perot resonances, gratings consisting of periodic arrays of grooves exhibit much higher SPP-excitation enhancement $\left(e_{\mathrm{SP}}(\max ) \approx 100\right)$; see Figs. 7 and 8 .

Although the main body of the study relies on fully vectorial computational results using the RCWA, all the results have been explained with a fully analytical model that provides closed-form expressions for the SPPexcitation rate. We expect that this study helps in providing an enhanced comprehension of the involvement of SPPs in the rich physics of periodic metallic surfaces. It may also help the design of future plasmonic devices, such as SPP generators, couplers, reflectors, etc.

\section{ACKNOWLEDGMENTS}

The authors would like to acknowledge helpful discussions with J.C. Rodier and J.P. Hugonin.

${ }^{\dagger}$ Present address: Institute of Materials Research and Engineering, 3 Research Link, Singapore 117602, Singapore.

\section{REFERENCES}

1. T. W. Ebbesen, H. F. Ghaemi, T. Thio, D. E. Grupp, and H. J. Lezec, "Extraordinary optical transmission through subwavelength hole arrays," Nature 391, 667-669 (1998).

2. M. Sobnack, W. Tan, N. Wanstall, T. Preist, and J. Sambles, "Stationary surface plasmons on a zero-order metal grating," Phys. Rev. Lett. 80, 5667-5670 (1998).

3. C. Genet and T. Ebbesen, "Light in tiny holes," Nature $\mathbf{4 4 5}$, 39-46 (2007).

4. J. V. Coe, J. M. Heer, S. Teeters-Kennedy, H. Tian, and K.
R. Rodriguez, "Extraordinary transmission of metal films with arrays of subwavelength holes," Annu. Rev. Phys. Chem. 59, 179-202 (2008).

5. W. L. Barnes, A. Dereux, and T. W. Ebbesen, "Surface plasmon subwavelength optics," Nature 424, 824-830 (2003).

6. E. Ozbay, "Plasmonics: merging photonics and electronics at nanoscale dimensions," Science 311, 189-193 (2006).

7. P. Lalanne and J. P. Hugonin, "Interaction between optical nano-objects at metallo-dielectric interfaces," Nat. Phys. 2, 551-556 (2006).

8. X. Y. Yang, H. T. Liu, and P. Lalanne, "Cross conversion between surface plasmon polaritons and quasicylindrical waves," Phys. Rev. Lett. 102, 153903 (2009).

9. H. Liu and P. Lalanne, "Microscopic theory of the extraordinary optical transmission," Nature 452, 728-731 (2008).

10. J. A. Porto, F. J. Garcia-Vidal, and J. B. Pendry, "Transmission resonances on metallic gratings with very narrow slits,” Phys. Rev. Lett. 83, 2845-2848 (1999).

11. Q. Cao and P. Lalanne, "Negative role of surface plasmons in the transmission of metallic gratings with very narrow slits," Phys. Rev. Lett. 88, 057403 (2002).

12. E. Popov, M. Nevière, S. Enoch, and R. Reinisch, "Theory of light transmission through subwavelength periodic hole arrays," Phys. Rev. B 62, 16100-16108 (2000).

13. B. Sturman, E. Podivilov, and M. Gorkunov, "Theory of extraordinary light transmission through arrays of subwavelength slits," Phys. Rev. B 77, 075106 (2008).

14. N. Garcia and M. Nieto-Vesperinas, "Theory of electromagnetic wave transmission through metallic gratings of subwavelength slits," J. Opt. A, Pure Appl. Opt. 9, 490-495 (2007).

15. A. I. Fernandez-Dominguez, F. J. Garcia-Vidal, and L. Martin-Moreno, "Resonant transmission of light through finite arrays of slits," Phys. Rev. B 76, 235430 (2007).

16. A. Vengurlekar, "Optical properties of metallo-dielectric deep trench gratings: role of surface plasmons and WoodRayleigh anomaly,” Opt. Lett. 33, 1669-1671 (2008).

17. D. Pacifici, H. Lezec, H. Atwater, and J. Weiner, "Quantitative determination of optical transmission through subwavelength slit arrays in Ag films: Role of surface wave interference and local coupling between adjacent slits," Phys. Rev. B 77, 115411 (2008)

18. M. Lu, X. Liu, L. Feng, J. Li, C. Huang, Y. Chen, Y. Zhu, S. Zhu, and N. Ming, "Extraordinary acoustic transmission through a 1D grating with very narrow apertures," Phys. Rev. Lett. 99, 174301 (2007).

19. H. Tamada, T. Doumuki, T. Yamaguchi, and S. Matsumoto, "Al wire-grid polarizer using the s-polarization resonance effect at the 0.8- $\mu \mathrm{m}$-wavelength band," Opt. Lett. 22, 419421 (1997).

20. A. Drezet, C. Genet, J. Laluet, and T. Ebbesen, "Optical chirality without optical activity: How surface plasmons give a twist to light," Opt. Express 16, 12559-12570 (2008).

21. P. Lalanne, J. Hugonin, H. Liu, and B. Wang, "A microscopic view of the electromagnetic properties of sub- $\lambda$ metallic surfaces," Surf. Sci. Rep. 64, 453-469 (2009).

22. A. Barbara, J. Le Perchec, S. Collin, C. Sauvan, J. L. Pelouard, T. López-Ríos, and P. Quémerais, "Generation and control of hot spots on commensurate metallic gratings," Opt. Express 16, 19127-19135 (2008).

23. S. Collin, G. Vincent, R. Haidar, N. Bardou, S. Rommeluere, and J. L. Pelouard, "Nearly perfect Fano transmission resonances through nanoslits drilled in a metallic membrane," Phys. Rev. Lett. 104, 027401 (2010).

24. A. Hessel and A. A. Oliner, "A new theory of Wood's anomalies on optical gratings," Appl. Opt. 4, 1275-1297 (1965).

25. P. Lalanne, J. P. Hugonin, and J. C. Rodier, "Approximate model for surface-plasmon generation at slit apertures," J. Opt. Soc. Am. A 23, 1608-1615 (2006).

26. B. Wang and P. Lalanne, "How many surface plasmons are locally excited on the ridges of metallic lamellar gratings," Appl. Phys. Lett. 96, 051115 (2010).

27. E. D. Palik, Handbook of Optical Constants of Solids (Academic, 1985). 
28. P. Lalanne, J. P. Hugonin, and P. Chavel, "Optical properties of deep lamellar gratings: A coupled Bloch-mode insight," J. Lightwave Technol. 24, 2442-2449 (2006).

29. M. G. Moharam, E. B. Grann, D. A. Pommet, and T. K. Gaylord, "Formulation for stable and efficient implementation of the rigorous coupled-wave analysis of binary gratings," J. Opt. Soc. Am. A 12, 1068-1076 (1995).

30. J. Liu, J. White, S. Fan, and M. Brongersma, "Side-coupled cavity model for surface plasmon-polariton transmission across a groove," Opt. Express 17, 17837-17848 (2009).

31. H. Liu, P. Lalanne, X. Yang, and J. P. Hugonin, "Surface plasmon generation by subwavelength isolated objects," IEEE J. Sel. Top. Quantum Electron. 14, 1522-1529 (2008).

32. C. Vassallo, Optical Waveguide Concepts (Elsevier, 1991).

33. J. C. Lagarias, J. A. Reeds, M. H. Wright, and P. E. Wright, "Convergence properties of the Nelder-Mead simplex method in low dimensions," SIAM J. Optim. 9, 112-147 (1998).
34. P. Lalanne, C. Sauvan, J. P. Hugonin, J. C. Rodier, and P. Chavel, "Perturbative approach for surface plasmon effects on flat interfaces periodically corrugated by subwavelength apertures," Phys. Rev. B 68, 125404 (2003).

35. J. L. Adams and L. C. Botten, "Double gratings and their applications as Fabry-Perot interferometers," J. Opt. 10, 109-117 (1979).

36. H. Lochbihler and R. Depine, "Highly conducting wire gratings in the resonance region," Appl. Opt. 32, 3459-3465 (1993).

37. H. Lochbihler, "Surface polaritons on gold-wire gratings," Phys. Rev. B 50, 4795-4801 (1994).

38. F. J. Garcia-Vidal and L. Martin-Moreno, "Transmission and focusing of light in one-dimensional periodically nanostructured metals," Phys. Rev. B 66, 155412 (2002).

39. W. Dai and C. M. Soukoulis, "Theoretical analysis of the surface wave along a metal-dielectric interface," Phys. Rev. B 80, 155407 (2009). 